\title{
Análise de robustez com o método Electre III: o caso da região de Campo das Vertentes em Minas Gerais
}

\author{
Robustness analysis with Electre III method: the case of the Campo \\ das Vertentes region in Minas Gerais
}

\author{
Carlos Eduardo Durange de Carvalho Infante ${ }^{1}$ \\ Fabricio Molica de Mendonça ${ }^{2}$ \\ Rogerio de Aragão Bastos do Valle ${ }^{1}$
}

\begin{abstract}
Resumo: Este estudo buscou aplicar o Electre III para classificar quatro aglomerados produtivos de micro e pequena empresa, localizados no Estado de Minas Gerais, em relação à capacidade de desenvolver estratégia de sobrevivência, por meio do relacionamento com o fornecedor, com o cliente ou distribuidor, com os concorrentes e com instituições públicas e privadas de apoio. O efeito conjunto dessas variáveis é que define a capacidade que eles possuem de desenvolver estratégias competitivas. Os resultados permitiram concluir que o agrupamento de produtores de artefatos em madeira de demolição é o que está mais articulado em relação ao conjunto de fatores de sucesso de um aglomerado, seguido pelos aglomerados de produtos em teares e em ferro, que se apresentam indiferentes entre si. $\mathrm{O}$ aglomerado de artefatos em estanho apresentou o último lugar, mostrando menor capacidade, por parte das empresas, em desenvolver estratégias conjuntas de sobrevivência. A Análise de Robustez foi realizada para garantir o resultado obtido com a aplicação, confirmando o ordenamento inicial.
\end{abstract}

Palavras-chave: Avaliação de Desempenho. Análise de Robustez. ELECTRE III. Agrupamento de MPEs.

\begin{abstract}
This study sought to apply the ELECTRE III to classify four production clusters of micro and small enterprises, located in Minas Gerais, according to the ability to develop a survival strategy through the relationship with the supplier, customer, distributor, competitors and public and private support companies. The combined effect of these variables defines the ability they have to develop competitive strategies. The results showed that the grouping of demolition wood manufacturers is the most appropriate regarding the success factors of a cluster, followed by clusters of and iron and weaving loom products, which apparently are not different from one another. The cluster of tin artifacts was the last one in the classification, showing a lower capacity for firms in developing joint strategies for survival. Robustness analysis was performed to confirm the results obtained with the application of ELECTRE III, confirming the initial ranking.
\end{abstract}

Keywords: Performance Evaluation. Robustness Analysis. ELECTRE III. MSE's cluster.

\section{Introdução}

Grande parte das atividades produtivas no Brasil é realizada por micro e pequenas empresas. Por um lado, tais empresas possuem a vantagem da flexibilidade em virtude de suas capacidades de se adaptar à dinâmica de mercado e, por outro lado, enfrentam a desvantagem da vulnerabilidade trazida pela difícil concorrência com empresas maiores. Nos últimos anos, tais empresas têm sido consideradas importantes para o desenvolvimento local e regional do País, por isso, é importante a realização de estudos voltados para o seu desenvolvimento.

No entanto, sabe-se que as micro e pequenas empresas podem conseguir sobrevida quando se agregam em aglomerados produtivos, a fim de auferir externalidades positivas geográficas e funcionais, que compreendem desde maior poder de barganha junto aos fornecedores e distribuidores até maior gerência sobre a forma de produção. Por meio da colaboração, conseguem auferir melhores resultados em virtude de melhores custos, melhor qualidade do produto, maior influência sobre os agentes econômicos e maior poder para enfrentar a concorrência.

Os aglomerados de micro e pequenas empresas podem conseguir sucesso, na medida em que: a) estreitam as relações com o mercado fornecedor, de modo a obter vantagens competitivas em relação a preços, prazos e quantidade de insumos; b) estreitam relações com o mercado comprador, para obter maiores informações sobre a demanda, estabelecer parcerias com clientes, praticar uma política de preço e prazo

\footnotetext{
${ }^{1}$ Universidade Federal do Rio de Janeiro - UFRJ, CEP 21941-972, Rio de Janeiro, RJ, Brasil, e-mail: infanteedu22@gmail.com

${ }^{2}$ Universidade Federal de São João del Rei - UFSJ, CEP 36301-160, São João del Rei, MG, Brasil, e-mail: fabriciomolica@ yahoo.com.br
} Recebido em 23/5/2012 — Aceito em 23/1/2014

Suporte financeiro: CAPES. 
compatível com a realidade da empresa; c) aumentam o nível de cooperação entre as empresas concorrentes, de modo que possam resolver problemas em comum e estabelecer trocas de informações saudáveis; e d) mantêm um sistema de proximidade com instituições públicas e privadas de apoio (CASAROTTO FILHO; PIRES, 2001; ABREU, 2002; MENDONÇA, 2010).

$\mathrm{O}$ efeito conjunto dessas variáveis sobre o aglomerado é o que garante maior ou menor grau de articulação dessas micro e pequenas empresas no sentido de desenvolver estratégias competitivas, garantindo a flexibilidade e produtividade e reduzindo a vulnerabilidade.

Com o intuito de analisar a eficiência desses aglomerados, se fazem necessários estudos na área de Auxílio Multicritério à Tomada de Decisão (AMD), visto que a análise conjunta das decisões em grupo requer uma metodologia robusta, que agregue todos os critérios fundamentais para a tomada de decisão. Entre os diversos modelos e metodologias desenvolvidos até então, merecem destaque os métodos da família ELECTRE, em decorrência de suas características fundamentais, dentre elas, a sobreclassificação de alternativas e, em especial, o método ELECTRE III (ROY, 1978), que se destina a tratar problemas de ordenação, introduzindo ponderações nos critérios adotados.

Este trabalho teve por finalidade classificar, por meio do método multicritério Electre III, o desempenho de quatro redes formadas por micro e pequenas empresas de base artesanal, localizadas na região Campo das Vertentes, no Estado de Minas Gerais, em função da capacidade de desenvolver estratégias competitivas, tendo como referência o efeito conjunto de variáveis linguísticas, ou seja, variáveis qualitativas, por exemplo, "muito bom", que serão transformadas em escalas quantitativas. Estas traduzem os quatro fatores já mencionados: a) grau de relacionamento com o fornecedor; b) grau de relacionamento com o cliente ou distribuidor; c) grau de relacionamento com os concorrentes localizados na mesma área geográfica; e d) grau de relacionamento com instituições públicas e privadas de apoio.

A escolha do método Electre III se justifica, pois foi originalmente desenvolvido por Roy (1978) para incorporar a natureza (imprecisa e incerta) fuzzy na ordenação de alternativas de melhor para pior, utilizando os limiares de indiferença e preferência. Assim, tal método se torna o mais apropriado para integrar diferentes variáveis linguísticas, de modo a selecionar a melhor dentre todas as alternativas, ou seja, a rede cujas empresas estão mais articuladas.

$\mathrm{O}$ artigo está estruturado da seguinte forma: a introdução, parte esta na qual se contextualiza a problemática central da pesquisa, assim como a metodologia que será utilizada. Na segunda parte, tem-se o referencial teórico, no qual, de acordo com a literatura, há uma integração de conceitos aliados à pesquisa de campo realizada. Nesta parte, também é detalhado o método utilizado, o Electre III, cujo valor matemático pode ser apreciado. Já na terceira parte, pode-se entender como foi realizada a pesquisa, de acordo com a aplicação do método escolhido e o passo a passo da aplicação de campo. Na quarta parte, tem-se a discussão da aplicação do método e os resultados. Na penúltima parte, antes da conclusão, foi realizada a análise de robustez, em que se propôs validar os resultados encontrados na fase anterior, complementada pela última parte, na qual se estudam os resultados desta análise. Por fim, a conclusão encerra o artigo, fechando os principais pontos abordados, garantindo, com efeito, os objetivos alcançados.

\section{Referencial teórico}

\subsection{Métodos de sobreclassificação - a família ELECTRE}

A sigla ELECTRE significa Eliminação e Escolha como expressão da realidade (Elimination et Choix Traduisant la Réalité). Consiste em uma família de métodos pertencentes à área de Auxílio Multicritério à Tomada de Decisão (AMD) que foi desenvolvida e aplicada, pela primeira vez, por Bernard Roy na década de 1960 com a finalidade de resolver um problema de escolha de melhor ação (alternativas) de um conjunto de ações, levando em consideração vários critérios que influenciavam na escolha. Logo, foi aplicado para resolver três problemas que envolvem a decisão: a escolha, a classificação e a ordenação (ROY, 1996). Hoje é considerada como o método mais robusto de análise que envolve multicritério.

Uma das características principais, que distingue o Electre de muitos outros múltiplos métodos de solução, é que ele é fundamentalmente um método não compensatório. Isto significa, em particular, que bons resultados em alguns critérios não podem compensar um ou mais resultados muito ruins em outros critérios. Outra característica do ELECTRE é que permite a incomparabilidade. Incomparabilidade - que não deve ser confundida com indiferença - ocorre entre algumas alternativas a e b, quando não há evidência clara a favor de algum tipo de preferência ou indiferença (ROY; BOUYSSOU, 1993).

Com o passar dos anos, o Electre, para atender às diferentes demandas para soluções de problemas multicritérios que foram surgindo, evoluiu para as versões I, II, III, IV, IS e TRI (árvore Electre) (FIGUEIRA et al., 2005). Todas essas versões são baseadas nos mesmos conceitos fundamentais, mas elas diferem tanto em termos operacionais quanto com o tipo de problema de decisão a ser resolvido. A versão I é usada para problemas de seleção. As 
versões II, III e IV para problemas de classificação e a versão TRI, para soluções de problemas de atribuição. Roy e Bouyssou (1993) especificam cada uma dessas versões, segundo eles:

a) A versão Electre I foi projetada para tratar os problemas de seleção por meio de relações de sobreclassificação a partir de índices de concordância e discordância, mediante comparação entre pares. Por meio dessa versão, dentre tantas alternativas, pode-se retirar a melhor, diante da atuação nos critérios selecionados. Esse método utiliza pesos para ordenar a importância dos critérios. Por exemplo, o trabalho realizado por Almeida e Costa (2003) buscou, por meio do Electra I, selecionar, dentro de um conjunto de equipamentos, aquele que apresenta melhor condição de uso, usando como critérios: riscos de segurança ao ser humano e às instalações, riscos ao meio ambiente e perdas de produção.

b) A versão Electre II foi um aprimoramento da versão I, usada para ordenar alternativas (classificação) a partir de um conjunto de índices de concordância e discordância associado à atribuição de pesos aos critérios. O intuito na utilização desse método não é escolher a melhor e sim classificá-las. Este método também utiliza pesos nos seus critérios. Como exemplo, pode-se citar a aplicação dessa versão por Costa, Motta e Gutierrez (2006) na solução de problemas de classificação de desempenho de docentes em relação à sua produção acadêmica.

c) A versão III tem por finalidade ordenar alternativas, como a versão II. No entanto, nessa versão, Roy (1978) incorporou a metodologia Fuzzy na construção do cálculo da ordenação de alternativas, permitindo a criação de pseudocritérios. A abordagem Fuzzy dessa versão permite a incorporação das imprecisões e incertezas do processo de tomada de decisões, fixando os limiares de preferência e indiferença. A ordenação de alternativas da melhor para a pior é conseguida por meio da introdução de ponderações nos critérios. Por exemplo, para solucionar o problema de ordenação de um conjunto de equipamentos em função dos critérios de risco de segurança, riscos ao meio ambiente e perdas de produção, por meio do Electra III, pode-se incorporar o ponto de vista do usuário, conforme foi usado por Freitas, Rubim e Manhães (2004). Outras aplicações bem-sucedidas dessa versão são encontradas em Roger, Bruen e Maystre (2000), Hokkanen e Salminen (1997), Al-Kloub, Al-Shemmeri e Pearman (1997), Georgopoulou, Lalas e Papagiannakis (1997) e Roger e Bruen (1998), em que se estudou o arranjo ótimo de resíduos sólidos, estratégias ambientais e planejamento energético.

d) A versão IV, assim como a versão III, tem como objetivo ordenar alternativas da melhor para a pior, no entanto, é empregada em problemas em que não se pode introduzir qualquer ponderação nos critério e/ou em que o agente de decisão não queira determinar pesos ou critérios. Dessa forma, obtém-se a solução por meio de uma sequência de relações de superação agrupadas. Essa versão foi criada para tratar, especificamente, o problema de planejamento urbano apresentado em Hugonnard e Roy (1984). No Brasil, por exemplo, Moreira (2007) aplicou esse método para avaliar projetos do SEBRAE. Outra aplicação interessante pode ser vista em Shanian e Savadogo (2006), que estudam placas bipolares de polímero de célula eletrolítica de membrana de combustível.

e) A versão IS pode ser considerada também uma evolução da versão I, no entanto, essa versão se diferencia pelo fato de permitir que sejam aplicados pesos aos critérios e, ainda, permite formulações Fuzzy.

f) A versão TRI tem como objetivo a classificação de alternativas, no entanto não as ordena, como pode ser visto nos estudos realizados por Figueira et al. (2005), Almeida-Dias, Figueira e Roy (2010) e Brito, Almeida e Mota (2010), nos quais se realizam estudos na área de reprodução assistida, ações referenciais e gás natural, respectivamente.

Então, percebe-se que, em problemas voltados para a construção de um ordenamento de alternativas, por meio de análise de relações de sobreclassificação fuzzy, em que é permitida a introdução de ponderação de critérios, conforme apresentado na introdução deste artigo, a versão mais indicada é o Electre III.

Tal versão depende da construção e exploração das relações de sobreclassificação das alternativas em duas fases, representadas na Figura 1, que são: a) construção de relações de classificação, em que as alternativas são emparelhadas e comparadas par a par, e classificadas dentro dos limiares dados pelo analista da decisão; e b) exploração das relações de classificação, em que as duas pré-classificações são construídas com dois procedimentos antagonistas (destilação ascendente e descendente). A combinação das duas pré-classificações resulta no resultado final. 


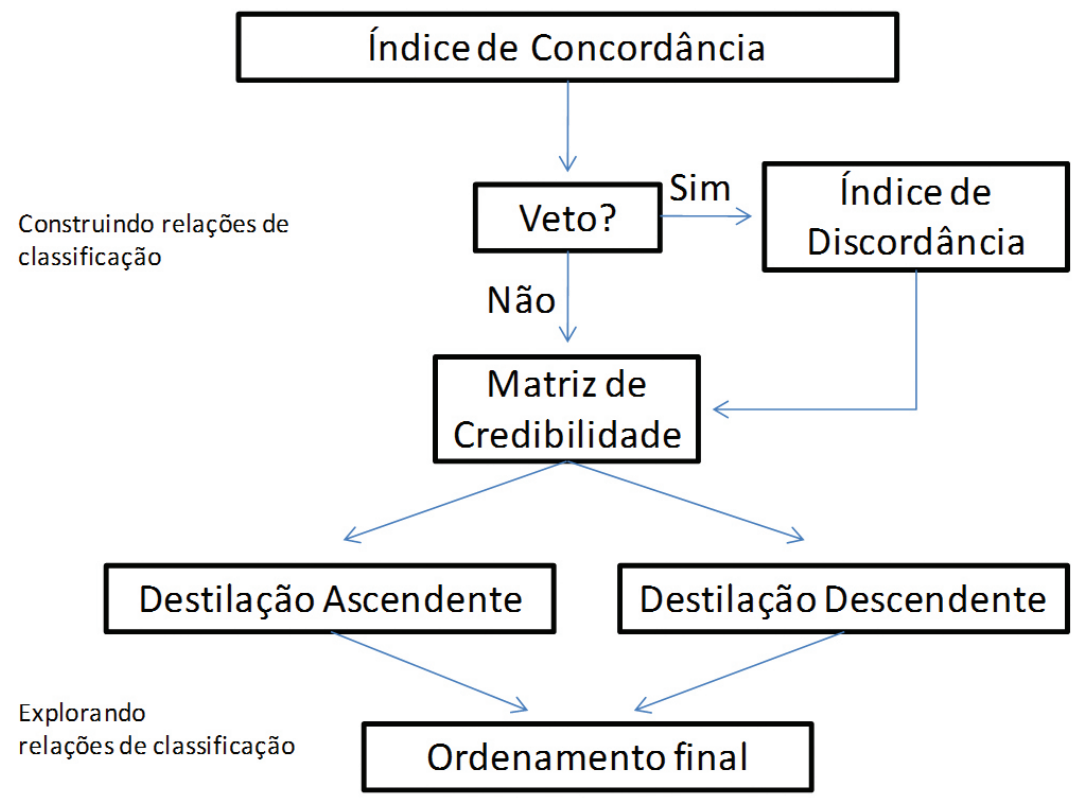

Figura 1. Fluxo do ELECTRE III. Fonte: Giannoulis e Ishizaka (2010).

\subsubsection{A construção de relações de classificação dentro do Electre III}

Os métodos de classificação tradicionais partem da relação de preferência e indiferença para compararem alternativas. Por exemplo, ao comparar duas alternativas "A" e "B", para se dizer que "A" supera "B", significa dizer que "A" é, pelo menos, tão bom quanto "B", entretanto, o desempenho de "A" é melhor, segundo critérios de preferência predefinidos. Assim, se assumirmos que existem critérios definidos (gj), para $\mathrm{j}=1,2,3 \ldots \mathrm{r}$, e dois conjuntos de alternativas $\mathrm{A}$ e $\mathrm{B}$, a modelagem de preferência tradicional assume as duas seguintes relações com as duas alternativas:

$$
\begin{aligned}
& \mathrm{aPb}(\mathrm{a} \text { é preferível } \mathrm{a} \mathrm{b}) \Leftrightarrow \mathrm{g}(\mathrm{a})>\mathrm{g}(\mathrm{b}) \\
& \mathrm{aIb}(\mathrm{a} \text { é indiferente } \mathrm{a} \mathrm{b}) \Leftrightarrow \mathrm{g}(\mathrm{a})=\mathrm{g}(\mathrm{b})
\end{aligned}
$$

Em cima do raciocínio dos métodos tradicionais, os métodos ELECTRE introduziram o conceito de limites de indiferença, q, que significam o limiar que uma alternativa pode transitar até ser indiferente à outra. Nesse caso, "A" é preferível a "B" quando "A" for tão boa quanto "B" e o seu desempenho for melhor segundo os critérios de preferência, considerando o limite de indiferença. Assim as relações de preferência são redefinidas como se segue:

$$
\begin{aligned}
\mathrm{aPb}(\mathrm{a} \text { é preferível a } \mathrm{b}) & \Leftrightarrow \mathrm{g}(\mathrm{a})>\mathrm{g}(\mathrm{b})+\mathrm{q} \\
\mathrm{alb}(\mathrm{a} \text { é indiferente } \mathrm{a} \mathrm{b}) & \Leftrightarrow|\mathrm{g}(\mathrm{a})-\mathrm{g}(\mathrm{b})| \leq \mathrm{q}
\end{aligned}
$$

Há um ponto em que um tomador de decisão parte de mudanças de indiferença para as de preferência estrita. Isso faz surgir uma boa razão para introduzir uma zona nebulosa entre indiferença e preferência estrita e, ainda, uma zona intermediária em que o tomador de decisão hesita entre preferência e indiferença. Esta zona de hesitação é referida como uma preferência fraca e também é uma relação binária, como P e I, e é modelada pela introdução de um limiar de preferência, p. Assim, tem-se um modelo de limiar duplo, com uma relação binária $\mathrm{Q}$, que são adicionais à medida de preferência fraca. Isto é:

$\mathrm{aPb}(\mathrm{a}$ tem preferência forte $\mathrm{a} b) \Leftrightarrow \mathrm{g}(\mathrm{a})-\mathrm{g}(\mathrm{b})>\mathrm{p}$

$\mathrm{aQb}(\mathrm{a}$ tem preferência fraca $\mathrm{a} b) \Leftrightarrow \mathrm{q}<\mathrm{g}(\mathrm{a})-\mathrm{g}(\mathrm{b}) \leq \mathrm{p}$

$\mathrm{alb}$ (a é indiferente $\mathrm{a} b$, e b é indiferente $\mathrm{a} a) \Leftrightarrow|\mathrm{g}(\mathrm{a})-\mathrm{g}(\mathrm{b})| \leq \mathrm{q}$

Embora a escolha do limiar adequado não seja fácil em decisões mais realistas, há boas razões para a escolha de valores diferentes de zero para P e Q.

Note-se que apenas foi considerado o caso mais simples, no qual os limiares P e Q são constantes, em oposição às funções de valor dos critérios, isto é, o caso dos limiares variáveis. Embora essa simplificação do uso de limiares constantes ajudarem a exposição do método ELECTRE, pode valer a pena usar limiares variáveis, no caso de o critério possuir valores maiores que podem elevar os limiares de preferência e indiferença.

Usando limites, o método ELECTRE busca construir uma relação de sobreclassificação aSb, em que $\mathrm{S}$ significa que, de acordo com o modelo global de preferências, há boas razões para considerar que "a alternativa A é pelo menos tão boa quanto B" ou “a não é pior do que b". Cada par de alternativas a e b é, então, testada para verificar se a afirmação aSb é válida ou não. Isto dá origem a uma das seguintes situações: 
Situação 1: aSb e não(bSa): "a" é preferível a "b" e não ("b" é preferível a "a");

Situação 2: não(aSb) e bSa: não ("a" é preferível a "b") e "b" é preferível a "a";

Situação 3: aSb e bSa: corresponde a uma situação de indiferença;

Situação 4: não(aSb) e não(bSa): corresponde a uma situação de incompatibilidade.

O teste para aceitar a afirmação aSb é implementado usando dois princípios:

i) um princípio de concordância, que exige que a maioria dos critérios, após considerar a sua importância relativa, seja a favor da afirmação $\mathrm{aSb}$ - o princípio da maioria; e

ii) o princípio de não discordância, que exige que a minoria dos critérios, que não suportam a afirmação, seja contra a afirmação - o respeito do princípio de minorias.

A implementação operacional desses dois princípios é hoje discutida, assumindo que todos os critérios devem ser maximizados. Primeiro, considere a relação de sobreclassificação definido para cada um dos r critérios, ou seja, aSjb, significa que "a é pelo menos tão boa quanto $b$ em relação ao j-ésimo critério", $\mathrm{j}=1,2, \ldots, \mathrm{r}$.

$\mathrm{O}$ critério j-ésimo está em concordância com a afirmação aSb se, e somente se, aSjb. Isto é, se gj(a) $\leq$ gj(b) - qj. Assim, mesmo que gj(a) seja inferior a gj(b) em um montante até qj, não viola a afirmação aSb e, com isso, permanece em concordância.

$\mathrm{O}$ critério j-ésimo está em discordância com a afirmação aSb se, e somente se, bPja. Isto é, se gj(b) $\geq \mathrm{gj}(\mathrm{a})$ - pj. Assim, se b é estritamente preferível a a no critério j, então é clara a não concordância com a afirmação aSb.

Com esses conceitos, já é possível medir a força da afirmação aSb. O primeiro passo é desenvolver uma medida de concordância, tal como consta $o$ índice de concordância $C(a, b)$, para cada par de alternativas $(\mathrm{a}, \mathrm{b}) \in \mathrm{A}$.

$$
\mathrm{C}_{\mathrm{j}}(\mathrm{a}, \mathrm{b})=\left\{\begin{array}{l}
1, \quad \operatorname{seg}_{\mathrm{j}}(\mathrm{a})+q_{\mathrm{j}} \geq \mathrm{g}_{\mathrm{j}}(\mathrm{b}) \\
0, \quad \operatorname{seg}_{\mathrm{j}}(\mathrm{a})+p_{\mathrm{j}} \leq \mathrm{g}_{\mathrm{j}}(\mathrm{b}), \mathrm{j}=1,2, \ldots, r \\
\frac{p(j)+g_{j}(a)-g_{j}(b)}{p_{j}-q_{j}}, \text { nos demais casos }
\end{array}\right.
$$

Em que,

C (a,b): índice de concordância das ações a e b;

$\mathrm{K}$ : soma dos pesos de todos os critérios;

$\mathrm{k}_{\mathrm{j}}$ : peso do critério $j$, para $j=1,2,3, \ldots, \mathrm{n}$;

$c_{\text {: }}$ : índice de concordância das ações a e b, sob o critério $j$.

Limiares e pesos representam entrada subjetiva do tomador de decisão. Os pesos utilizados no modelo ELECTRE são bastante diferentes das ponderações utilizadas em outras abordagens de modelagem de decisão, como a decisão de abordagem analítica (SMART) de Edwards (1997). Na decisão de modelos analíticos, por exemplo, os pesos são as taxas de substituição e avaliam a preferência relativa entre os critérios. Os pesos no ELECTRE são "coeficientes de importância" e, como Vincke (1992) aponta, eles são como votos dados a cada um dos critérios. Roger, Bruen e Maystre (2000) dizem que os regimes de opinião existentes prestam uma útil discussão do conceito de ponderação em ELECTRE. Cuidados também devem ser tomados na determinação dos valores limite, que deve incidir especificamente para cada critério e refletem a preferência de cada decisor. Procedimentos para a escolha dos limiares adequados são abordados por Roger e Bruen (1998).

Até o momento, não foi tido em conta o princípio de discordância. No índice de concordância, temos uma medida da extensão em que estamos em harmonia com a afirmação de que "a é pelo menos tão boa quanto b". Para calcular a discordância, um limiar mais conhecido é o limiar de veto. O limiar de veto vj permite que a afirmação aSb seja recusada totalmente por todos os critérios j, gj(b) > gj(a) + vj. O índice de discordância para cada critério $\mathrm{j}$, dj(a,b) é calculado como mostrado a seguir:

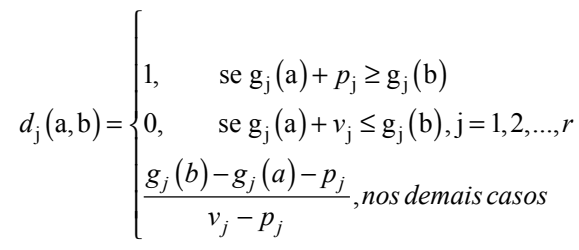

Em que:

$\mathrm{z}_{\mathrm{i}}(\mathrm{X})$ : desempenho da alternativa $\mathrm{X}$ no critério i; $p_{i}$ : limiar de preferência da alternativa no critério i; $v_{i}$ : limiar de veto da alternativa no critério $i$.

\subsubsection{A construção de um modelo para medir o grau de sobreclassificação}

Para cada par de alternativas (a,b), há uma medida de concordância e uma de discordância. O passo final na fase de construção do modelo é combinar estas duas medidas para produzir uma medida do grau de sobreclassificação, ou seja, um índice de credibilidade que avalia a força da afirmação "a é pelo menos tão boa quanto b". O grau de credibilidade para cada par de alternativas $(\mathrm{a}, \mathrm{b}) \in \mathrm{A}$ é definido como:

$$
\mathrm{S}(\mathrm{a}, \mathrm{b})=\left\{\begin{array}{l}
\mathrm{C}(\mathrm{a}, \mathrm{b}), \mathrm{se}_{\mathrm{j}}(\mathrm{a}, \mathrm{b}) \leq \mathrm{C}(\mathrm{a}, \mathrm{b}) \\
\mathrm{C}(\mathrm{a}, \mathrm{b}) * \prod_{j \in J(\mathrm{a}, \mathrm{b})} \frac{1-d_{j}(a, b)}{1-C(a, b)}, \\
\text { onde } \mathrm{J}(\mathrm{a}, \mathrm{b}) \text { é o conjunto de critérios que } \\
\text { satisfazem dj }(\mathrm{a}, \mathrm{b})>\mathrm{C}(\mathrm{a}, \mathrm{b})
\end{array}\right.
$$

Em que:

$\mathrm{J}(\mathrm{A}, \mathrm{B})$ : é o conjunto de critérios para que $\mathrm{D}_{\mathrm{i}}(\mathrm{A}, \mathrm{B})>\mathrm{C}(\mathrm{A}, \mathrm{B})$. 
Essa fórmula presume que, se a força da concordância ultrapassa o da discordância, o valor de concordância não deve ser modificado. Caso contrário, é forçado a questionar a afirmação de que o aSb e modificar $\mathrm{C}(\mathrm{a}, \mathrm{b})$ de acordo com a equação acima. Se a discordância é de 1,0 para qualquer $(\mathrm{a}, \mathrm{b}) \mathrm{A}$ e qualquer critério $\mathrm{j}$, então não se tem confiança de que $\mathrm{aSb}$, portanto, $\mathrm{S}(\mathrm{a}, \mathrm{b})=0,0$. Isto conclui a construção do modelo de sobreclassificação.

\subsection{Variáveis linguísticas usadas e o método Electre III}

Variáveis linguísticas são variáveis cujos valores são palavras em linguagem natural representadas em conjuntos difusos, usadas para manipular problemas imprecisos e complexos (ZADEH, 1975).

Por exemplo, "peso" é uma variável linguística e seus valores são: "muito leve", "leve", "médio", "alto", "muito alto", etc. (LIANG; WANG, 1991). Uma área de aplicação particularmente importante das variáveis linguísticas é a do raciocínio que não é "quase muito preciso" e "não muito impreciso", chamado raciocínio aproximado (PEREIRA, 1995).

Segundo Oliveira Júnior (1999), as variáveis linguísticas se apresentam como os "tijolos" das proposições difusas, uma vez que: a) possuem conteúdo variável como em uma linguagem de programação; b) assumem valores linguísticos (alto, baixo, médio, quente) representados por conjuntos difusos; e c) possuem identificação nominal.

Percebe-se que o conceito de variável linguística é muito útil para lidar com situações que são muito complexas ou mal definidas para serem racionalmente descritas nas expressões quantitativas convencionais. Por isso, tais variáveis devem ser tratadas por meio de métodos capazes de incorporar a natureza difusa de tais variáveis.

O método Electra III foi desenvolvido por Roy (1978) para incorporar a natureza imprecisa e incerta na ordenação de alternativas de melhor para pior, utilizando os limiares de indiferença e preferência. Sendo, portanto, o mais indicado para integrar diferentes variáveis linguísticas dentro de diferentes alternativas e, ainda, classificar e selecionar a melhor dentre tais alternativas.

\section{Metodologia}

Para aplicar o método multicritério Electre III em um problema de classificar o desempenho de redes de micro e pequenas empresas, foram selecionados quatro agrupamentos de micro e pequenas empresas, localizados na região Campo das Vertentes, no Estado de Minas Gerais, a saber: a) um agrupamento de produtores de artigos em estanho (G1); b) um agrupamento de produtores de artigos elaborados em teares $(\mathrm{G} 2)$; c) um agrupamento de produtores de artigos em ferro (G3); e d) um agrupamento de produtores de artigos em madeira de demolição (G4). Cada agrupamento foi considerado uma alternativa, formando assim um grupo de alternativas $(\mathrm{G} 1, \mathrm{G} 2$, G3 e G4).

A classificação de cada alternativa foi feita em função de quatro critérios, apresentados como: a) grau de relacionamento com o fornecedor (V1); b) grau de relacionamento com o cliente ou distribuidor (V2); c) grau de relacionamento com os concorrentes localizados na mesma área geográfica (V3); e d) grau de relacionamento com instituições públicas e privadas de apoio (V4). Estes critérios foram selecionados mediante importância para os agrupamentos selecionados, tendo em vista a atuação das micro e pequenas empresas.

Assim se formou o conjunto de critérios (V1, V2, $\mathrm{V} 3$ e V4). A coleta de informações se deu por meio de uma entrevista, acompanhada de questionários, aplicados a dez proprietários pertencentes às empresas de cada agrupamento, de modo a levantar a importância de cada critério (V1, V2, V3 e V4) na visão de cada proprietário. Por isso, foram usadas as variáveis linguísticas descritas na Tabela 1 . As demais variáveis linguísticas usadas para a descrição do desempenho das alternativas estão descritas na Tabela 2.

\subsection{Resultados dos questionários}

Os pesos linguísticos dados, por meio dos questionários aplicados, pelos integrantes de cada agrupamento, no que se refere ao relacionamento com os fornecedores, podem ser visualizados na Tabela 2 .

Os pesos linguísticos dados, por meio de questionários aplicados, pelos integrantes de cada agrupamento, no que se refere ao relacionamento com os clientes ou distribuidores, pode ser visualizado na Tabela 3.

Os pesos linguísticos dados, por meio de questionários aplicados, pelos integrantes de cada agrupamento, no que se refere ao relacionamento com os concorrentes localizados na mesma área geográfica, pode ser visualizado na Tabela 4 .

Tabela 1. Pesquisa qualitativa versus pesquisa quantitativa.

\begin{tabular}{lc}
\hline \multicolumn{1}{c}{ Variável Linguística } & Peso \\
\hline Muito Pobre & 0 \\
Entre muito pobre e pobre & 0 \\
Pobre & 0 \\
Entre pobre e razoável & 0 \\
Entre razoável e bom & 0,3 \\
Bom & 0,6 \\
Entre bom e muito bom & 0,6 \\
Muito Bom & 0,8 \\
\hline
\end{tabular}

Fonte: Dados da Pesquisa.. 
Os pesos linguísticos dados, por meio de questionários aplicados, pelos integrantes de cada agrupamento, no que se refere ao grau de relacionamento com instituições públicas e privadas de apoio (V4), pode ser visualizado na Tabela 5.

Os pesos de cada critério, inicialmente, serão os valores máximos atribuídos em cada questinário realizado.

\subsection{Aplicação do ELECTRE III}

Para a construção da Matriz de Desempenho, cada variável linguística ( $\mathrm{MB}, \mathrm{A}, \mathrm{M}, \mathrm{B}, \mathrm{MB})$ foi relacionada a uma escala quantitativa, dentro do intervalo $[0,1]$
(Tabela 6). Estes valores foram atribuídos pelos analistas de decisão, ou seja, pesquisadores.

Depois de selecionar os pesos dos critérios e os respectivos valores quantitativos das variáveis linguísticas, foi necessário verificar e atribuir os limiares de preferência, indiferença e veto (Tabela 7).

Como todas as alternativas podem satisfazer a afirmação aSb (a alternativa "a" é tão boa quanto a alternativa "b"), optou-se por não atribuir valores ao limiar de veto. Assim, os respectivos valores dos limiares foram atribuídos pelos analistas de decisão, aqueles submetidos aos questionários, e eles se referem ao intervalo garantido da alternativa

Tabela 2. Pesos linguísticos dados pelos integrantes de cada agrupamento, no que se refere ao relacionamento com o fornecedor.

\begin{tabular}{|c|c|c|c|c|c|c|c|c|c|c|c|c|c|}
\hline Agrupamentos & $Q_{1}$ & $\mathbf{Q}_{2}$ & $\mathbf{Q}_{3}$ & $\mathbf{Q}_{4}$ & $Q_{5}$ & $Q_{6}$ & $\mathbf{Q}_{7}$ & $\mathbf{Q}_{8}$ & $Q_{9}$ & $Q_{10}$ & média & Máx (V1) & Mín(V1) \\
\hline Setor de Estanho $\left(\mathbf{G}_{\mathbf{1}}\right)$ & 0 & 0 & 0 & 0 & 0 & 0 & 0 & 0 & 0 & 0 & 0,15 & 0,60 & 0,15 \\
\hline Setor de Tear $\left(\mathbf{G}_{2}\right)$ & 0 & 0 & 1 & 1 & 0 & 0 & 1 & 1 & 0 & 0,30 & 0,42 & & \\
\hline $\begin{array}{l}\text { Setor de Madeira de } \\
\text { Demoliçãoo }\left(\mathbf{G}_{\mathbf{3}}\right)\end{array}$ & 1 & 1 & 1 & 1 & 0 & 1 & 1 & 1 & 1 & 0,60 & 0,57 & & \\
\hline Setor de Ferro $\left(\mathbf{G}_{4}\right)$ & 1 & 1 & 1 & 1 & 1 & 1 & 1 & 1 & 1 & 0,60 & 0,60 & & \\
\hline
\end{tabular}

Fonte: Dados da pesquisa.

Tabela 3. Pesos linguísticos dados pelos integrantes de cada agrupamento, no que se refere ao relacionamento com o cliente ou o distribuidor.

\begin{tabular}{|c|c|c|c|c|c|c|c|c|c|c|c|c|c|}
\hline Agrupamentos & $\mathbf{Q}_{1}$ & $\mathbf{Q}_{2}$ & $\mathbf{Q}_{3}$ & $\mathbf{Q}_{4}$ & $\mathbf{Q}_{5}$ & $\mathbf{Q}_{6}$ & $\mathbf{Q}_{7}$ & $\mathbf{Q}_{8}$ & $\mathbf{Q}_{9}$ & $\mathbf{Q}_{10}$ & média & Máx(V2) & Mín(V2) \\
\hline Setor de Estanho $\left(\mathbf{G}_{\mathbf{1}}\right)$ & 0 & 0 & 0 & 1 & 1 & 0 & 1 & 1 & 1 & 0,30 & 0,45 & 0,60 & 0,30 \\
\hline Setor de Tear $\left(\mathbf{G}_{\mathbf{2}}\right)$ & 1 & 1 & 1 & 1 & 1 & 1 & 1 & 1 & 1 & 0,60 & 0,60 & & \\
\hline $\begin{array}{l}\text { Setor de Madeira de } \\
\text { Demolição }\left(\mathbf{G}_{\mathbf{3}}\right)\end{array}$ & 1 & 1 & 1 & 1 & 1 & 1 & 1 & 1 & 1 & 0,60 & 0,60 & & \\
\hline Setor de Ferro $\left(\mathbf{G}_{4}\right)$ & 0 & 0 & 0 & 0 & 0 & 0 & 0 & 0 & 0 & 0,30 & 0,30 & & \\
\hline
\end{tabular}

Fonte: Dados da pesquisa.

Tabela 4. Pesos linguísticos dados pelos integrantes de cada agrupamento, no que se refere ao relacionamento com o cliente ou o distribuidor.

\begin{tabular}{|c|c|c|c|c|c|c|c|c|c|c|c|c|c|}
\hline Agrupamentos & $Q_{1}$ & $\mathbf{Q}_{2}$ & $Q_{3}$ & $\mathbf{Q}_{4}$ & $Q_{5}$ & $Q_{6}$ & $\mathbf{Q}_{7}$ & $\mathbf{Q}_{8}$ & $\mathbf{Q}_{9}$ & $Q_{10}$ & média & Máx(V3) & Mín(V3) \\
\hline Setor de Estanho $\left(\mathbf{G}_{\mathbf{1}}\right)$ & 0 & 0 & 0 & 0 & 0 & 0 & 0 & 0 & 0 & 0 & 0 & 0,48 & 0 \\
\hline Setor de Tear $\left(\mathbf{G}_{\mathbf{2}}\right)$ & 1 & 0 & 1 & 1 & 0 & 0 & 1 & 1 & 1 & 0,30 & 0,48 & & \\
\hline $\begin{array}{l}\text { Setor de Madeira de } \\
\text { Demolição }\left(\mathbf{G}_{\mathbf{z}}\right)\end{array}$ & 0 & 0 & 1 & 1 & 0 & 0 & 1 & 1 & 1 & 0,30 & 0,45 & & \\
\hline Setor de Ferro $\left(\mathbf{G}_{\mathbf{4}}\right)$ & 0 & 0 & 0 & 0 & 0 & 0 & 0 & 0 & 0 & 0 & 0 & & \\
\hline
\end{tabular}

Fonte: Dados da pesquisa.

Tabela 5. Pesos linguísticos dados pelos integrantes de cada agrupamento, no que se refere ao relacionamento com o cliente ou o distribuidor.

\begin{tabular}{|c|c|c|c|c|c|c|c|c|c|c|c|c|c|}
\hline Agrupamentos & $Q_{1}$ & $Q_{2}$ & $\mathbf{Q}_{3}$ & $\mathbf{Q}_{4}$ & $Q_{5}$ & $Q_{6}$ & $\mathbf{Q}_{7}$ & $\mathbf{Q}_{8}$ & $Q_{9}$ & $Q_{10}$ & média & Máx(V4) & Mín(V4) \\
\hline Setor de Estanho $\left(\mathbf{G}_{\mathbf{1}}\right)$ & 1 & 1 & 1 & 1 & 1 & 1 & 1 & 1 & 1 & 0,60 & 0,66 & 0,66 & 0,06 \\
\hline Setor de Tear $\left(\mathbf{G}_{\mathbf{2}}\right)$ & 0 & 0 & 0 & 0 & 0 & 0 & 0 & 0 & 0 & 0,30 & 0,06 & & \\
\hline $\begin{array}{l}\text { Setor de Madeira de } \\
\text { Demolição }\left(\mathbf{G}_{\mathbf{3}}\right)\end{array}$ & 0 & 0 & 0 & 0 & 0 & 0 & 0 & 0 & 0 & 0,30 & 0,21 & & \\
\hline Setor de Ferro $\left(\mathbf{G}_{\mathbf{4}}\right)$ & 1 & 1 & 1 & 1 & 0 & 0 & 1 & 1 & 0 & 0,60 & 0,51 & & \\
\hline
\end{tabular}

Fonte: Dados da pesquisa. 
a sobre a alternativa b, no critério j. Por exemplo, um limiar de indiferença (q) de 0,1, significa que para determinado critério j tem-se o limiar de aceitação de $10 \%$ de uma alternativa sobre a outra. No estudo realizado, um limiar de indiferença resultará em melhor análise pairwise das alternativas propostas.

\section{Discussão da aplicação do método ELECTRE III}

A partir dos dados coletados, por meio das entrevistas, com base em questionários, aplicados a dez empresários de cada agrupamento, os dados foram compilados por meio do uso da média aritmética das opiniões, construindo, assim, a matriz de desempenho (Tabela 8). Nessa matriz, são representadas as alternativas e os pesos atribuídos às variáveis linguísticas e seus respectivos limiares, dentro dos critérios definidos.

A partir da aplicação da Equação 1, do método Electre III, foi possível a comparação par a par das alternativas, analisando-as segundo a afirmação aSb. Com isso, foi possível obter a Matriz de Concordância (Tabela 9). Por meio dessa tabela, pôde-se perceber que a alternativa $\mathrm{G} 2$ é $100 \%$ melhor que a alternativa G1, A alternativa G1 não é referencial a nenhuma outra. A alternativa G2 é apenas $25 \%$ melhor que a alternativa $\mathrm{G} 4$, o que mostra a prevalência da última (G4) em relação à primeira (G2).

Como não se optou por utilizar o limiar de veto, a matriz de credibilidade fica semelhante à matriz de concordância. Ao considerar a matriz de concordância na Tabela 4, a ordem final pode ser representada por meio do Quadro 1.

Por meio da análise dessa tabela, pode-se perceber que: a) a alternativa G1 não é preferível às alternativas; b) a alternativa $\mathrm{G} 2$ é preferível à $\mathrm{G} 1$, indiferente à $\mathrm{G} 3$ e não preferível à G4; c) a alternativa G3 é preferível à $\mathrm{G} 1$, indiferente à $\mathrm{G} 2$ e não preferível à $\mathrm{G} 4$; d) a alternativa G4 é preferível a todas as alternativas.

Interpretando esse resultado, pode-se afirmar que, por meio do resultado apresentado pelo Electra III, o agrupamento de produtores de artefatos em madeira de demolição (alternativa G4) é o que está mais articulado, em relação ao conjunto de fatores de sucesso de um aglomerado, considerados como os seguintes critérios selecionados: a) grau de relacionamento com o fornecedor; b) grau de relacionamento com o cliente ou distribuidor; c) grau de relacionamento com os concorrentes localizados na mesma área geográfica; e d) grau de relacionamento com instituições públicas e privadas de apoio.

As aglomerações de artigos elaborados em teares (G2) e o agrupamento de produtores de artigos em ferro (G3) estão menos articulados do que o de artefatos em madeira de demolição. No entanto, esses dois aglomerados apresentam indiferença relativa entre eles, podendo afirmar que, no conjunto de critérios, apresentaram o mesmo resultado. Tais aglomerados necessitam melhorar o grau de relacionamento com o fornecedor e com o cliente ou distribuidor, bem como com as instituições públicas e privadas de apoio.

$\mathrm{O}$ aglomerado de produtores de artefatos em estanho (G1) é o que está menos articulado, ou seja, é aquele em que as empresas apresentam a menor capacidade de desenvolver estratégias conjuntas de sobrevivência.

Tabela 6. Pesquisa qualitativa versus pesquisa quantitativa.

\begin{tabular}{cc}
\hline Variável Linguística & Peso \\
\hline Muito Alto & 1 \\
Alto & 0,75 \\
Médio & 0,5 \\
Baixo & 0,25 \\
Muito Baixo & 0 \\
\hline
\end{tabular}

Fonte: Dados da Pesquisa..

Tabela 7. Limiares de preferência, indiferença e veto.

\begin{tabular}{lcccc}
\hline \multirow{2}{*}{\multicolumn{1}{c}{ Limiares }} & \multicolumn{4}{c}{ Critérios } \\
\cline { 2 - 5 } & V1 & V2 & V3 & V4 \\
\hline Limite de Indiferença (q) & 0,1 & 0,1 & 0,1 & 0,1 \\
Limite de preferência (p) & 0,2 & 0,2 & 0,2 & 0,2 \\
Veto & - & - & - & - \\
\hline
\end{tabular}

Fonte: Dados da pesquisa.

Tabela 8. Matriz de Desempenho das alternativas versus critérios.

\begin{tabular}{ccccc}
\hline \multirow{2}{*}{ Alternativas } & \multicolumn{4}{c}{ Critérios } \\
\cline { 2 - 5 } & V1 & V2 & V3 & V4 \\
\hline G1 & 0,25 & 0,25 & 0,75 & 0,50 \\
G2 & 0,50 & 0,25 & 1 & 0,75 \\
G3 & 0,50 & 0,50 & 1 & 0,75 \\
G4 & 0,50 & 0,75 & 1 & 1 \\
\hline
\end{tabular}

Fonte: Dados da pesquisa.

Tabela 9. Matriz de Concordância entre as alternativas.

\begin{tabular}{ccccc}
\hline & G1 & G2 & G3 & G4 \\
\hline G1 & 1 & 0 & 0 & 0 \\
G2 & 1 & 1 & 1 & 0,21 \\
G3 & 1 & 1 & 1 & 0,21 \\
G4 & 1 & 1 & 1 & 1 \\
\hline
\end{tabular}

Fonte: Dados da pesquisa.

Quadro 1. Matriz de Pré-ordem final.

\begin{tabular}{|c|c|c|c|c|}
\hline & G1 & G2 & G3 & G4 \\
\hline G1 & I & $\mathrm{P}^{-}$ & $\mathrm{P}^{-}$ & $\mathrm{P}^{-}$ \\
\hline G2 & $\mathrm{P}$ & $\mathrm{I}$ & $\mathrm{I}$ & $\mathrm{P}^{-}$ \\
\hline $\mathrm{G} 3$ & $\mathrm{P}$ & $\mathrm{I}$ & $\mathrm{I}$ & $\mathrm{P}^{-}$ \\
\hline $\mathrm{G} 4$ & $\mathrm{P}$ & $\mathrm{P}$ & $\mathrm{P}$ & $\mathrm{I}$ \\
\hline
\end{tabular}

Fonte: Dados da pesquisa. 
A inserção dessas empresas em aglomerado pouco contribui para o aumento da sobrevivência, pois apenas se encontram geograficamente próximas, deixando de aproveitar as externalidades provenientes da aglomeração. Por isso, nesse aglomerado, são necessários trabalhos de fortalecimento dentro dos critérios ou fatores estudados.

A classificação final das alternativas pode ser vista por meio da Figura 2. Esta classificação mostra o ordenamento segundo os critérios selecionados. Visualiza-se a hierarquia na tomada de decisão estratégica.

\section{Análise de sensibilidade, estabilidade e robustez}

Para a empresa, talvez não seja interessante apenas ter a melhor decisão no momento, mas também conhecer as alternativas possíveis de decisão. A

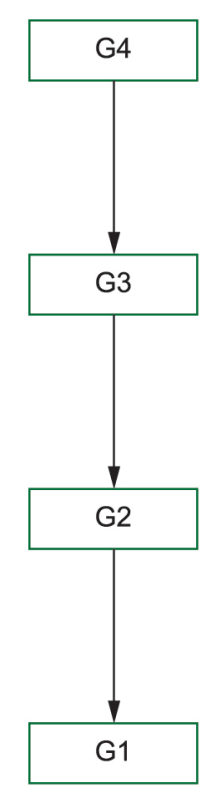

Figura 2. Grafo Final das alternativas. Fonte: Dados da pesquisa. empresa poderia contentar-se com uma boa decisão dentro de suas possibilidades ou com a segunda melhor opção (SHIMIZU, 2006).

Gomes, Gomes e Almeida (2002) indicam que, após a aplicação de um método multicritério, torna-se importante a realização de uma análise de sensibilidade, com o intuito de verificar de que forma as variações introduzidas nos parâmetros característicos do método influenciam os resultados obtidos. Já Gomes (2007) coloca a análise de sensibilidade como a última fase do processo de tomada de decisão, evidenciando mais ainda sua importância. Os autores afirmam que uma análise de estabilidade tem por objetivo verificar a velocidade com que uma solução se degrada em um nível pré-determinado.

Já a análise de robustez tem por objetivo verificar até que ponto, após análise de sensibilidade, a pré-ordem encontrada no conjunto de soluções não dominadas não se altera (GOMES; GOMES; ALMEIDA, 2002).

\section{Resultados das análises de robustez no ELECTRE III}

A análise de robustez foi realizada variando-se os parâmetros dos pesos, verificando desta maneira se houve variações significativas na classificação final. A Tabela 10 sumariza esta análise.

Observa-se pela tabela que, para todos os critérios, a classificação das alternativas não se altera, exceto no critério V2, no qual há a indiferença entre as alternativas $\mathrm{G} 2$ e G3, concluindo-se, neste caso, que uma variação de $50 \%$ no peso atribuído modifica o resultado da classificação. Esta análise de robustez possibilita classificar a solução encontrada como "Sensibilidade Fraca", em vista dos resultados que se modificam com a alteração dos parâmetros.

\section{Conclusão}

O trabalho teve por finalidade classificar (ordenar), por meio do método multicritério de avaliação de desempenho Electre III, o desempenho de quatro agrupamentos (redes) de micro e pequenas empresas

Tabela 10. Resultado da variação dos pesos dos critérios.

\begin{tabular}{|c|c|c|c|c|c|c|c|}
\hline \multirow{2}{*}{ Critério } & \multirow{2}{*}{$\begin{array}{c}\text { Peso } \\
\text { Original }\end{array}$} & \multirow{2}{*}{$\begin{array}{c}\text { Peso } \\
\text { Médio }\end{array}$} & \multirow{2}{*}{$\begin{array}{l}\text { Peso } \\
\text { Mín }\end{array}$} & \multicolumn{4}{|c|}{ Classificação } \\
\hline & & & & 1 & 2 & 3 & 4 \\
\hline \multirow{2}{*}{ V1 } & \multirow{2}{*}{0,60} & 0,43 & - & G4 & G3 & G2 & G1 \\
\hline & & - & 0,15 & G4 & G3 & $\mathrm{G} 2$ & G1 \\
\hline \multirow{2}{*}{ V2 } & \multirow{2}{*}{0,60} & 0,48 & - & G4 & G3 & G2 & G1 \\
\hline & & - & $\mathbf{0 , 3 0}$ & G4 & G2 / G3 & - & G1 \\
\hline \multirow{2}{*}{ V3 } & \multirow{2}{*}{0,48} & 0,23 & - & G4 & G3 & G2 & G1 \\
\hline & & - & 0,00 & G4 & G3 & $\mathrm{G} 2$ & G1 \\
\hline \multirow{2}{*}{ V4 } & \multirow{2}{*}{0,66} & 0,36 & - & G4 & G3 & G2 & G1 \\
\hline & & - & 0,06 & G4 & G3 & G2 & G1 \\
\hline
\end{tabular}

Fonte: Dados da pesquisa. 
produtoras de artesanato, localizadas na região Campo das Vertentes, no Estado de Minas Gerais, a saber: um agrupamento de produtores de artefatos em estanho; um agrupamento de produtores de artefatos em tear; um agrupamento de produtores de artefatos em ferro; e um agrupamento de produtores de artefatos em madeira de demolição. Cada agrupamento foi identificado como alternativas G1, G2, G3 e G4, respectivamente.

O estudo partiu do princípio de que um aglomerado consegue sobrevida quando consegue boa articulação conjunta das variáveis: a) grau de relacionamento com o fornecedor; b) grau de relacionamento com o cliente ou distribuidor; c) grau de relacionamento com os concorrentes localizados na mesma área geográfica; e d) grau de relacionamento com instituições públicas e privadas de apoio. Cada variável foi considerada um critério, identificados como critérios V1, V2, V3 e V4, respectivamente.

A partir da aplicação, a cada critério, das variáveis linguísticas (Muito Alto, Alto, Médio, Baixo e Muito Baixo) obtidas por meio de respostas dadas por dez entrevistados, em cada aglomerado, pôde-se construir uma matriz de desempenho, dentro dos limiares de preferência, indiferença e veto, que, ao introduzir os seus resultados nas fórmulas do Electre III, permitiu a criação da matriz de concordância entre alternativas e ordenamento final.

Os resultados obtidos por meio da matriz de concordância permitiram concluir que o agrupamento de produtores de artefatos em madeira de demolição é o que está mais articulado em relação ao conjunto de fatores de sucesso de um aglomerado, dentro dos critérios preestabelecidos. As aglomerações de artigos elaborados em teares e o agrupamento de produtores de artigos em ferro se apresentam em segundo lugar. $\mathrm{O}$ aglomerado de artefatos em estanho apresentou o último lugar, mostrando menor capacidade, por parte das empresas, em desenvolver estratégias conjuntas de sobrevivência.

A análise de robustez foi importante para a comprovação da classificação final das alternativas.

A contribuição teórica, neste trabalho, foi de fundamental importância para o entendimento do conceito de aglomerados industriais, assim como a análise multicritério de apoio à decisão. A revisão bibliográfica realizada permitiu integrar a metodologia aplicada aos teóricos lidos.

\section{Agradecimentos}

Apoio financeiro da Coordenação de Aperfeiçoamento de Pessoal do Nível Superior - CAPES.

\section{Referências}

ABREU, J. C. de. Estratégia e oportunidades locais: um estudo sobre rede dinâmica em aglomerados de empreendedores de base artesanal. 2002. Tese (Doutorado) - Instituto Alberto Luiz Coimbra de Pós-Graduação e Pesquisa de Engenharia, Universidade Federal do Rio de Janeiro - UFRJ, Rio de Janeiro, 2002.

AL-KLOUB, B.; AL-SHEMMERI, T.; PEARMAN, A. The role of weights in multi-criteria decision aid, and the ranking of water projects in Jordan. European Journal of Operational Research, v. 99, n. 2, p. 278-288, 1997. http://dx.doi.org/10.1016/S0377-2217(96)00051-3

ALMEIDA, A. T.; COSTA, A. P. C. S. Aplicações com métodos multicritério de apoio à decisão. Recife: Universitária UFPE, 2003.

ALMEIDA-DIAS, J.; FIGUEIRA, J. R.; ROY, B. ELECTRE TRI-C: a multiple criteria sorting method based on characteristic reference actions. European Journal of Operational Research, v. 204, n. 13, p. 565-580, 2010. http://dx.doi.org/10.1016/j.ejor.2009.10.018

BRITO, A. J.; ALMEIDA, A. T.; MOTA, C. M. M. A multicriteria model for risk sorting of natural gas pipelines based on ELECTRE TRI integrating utility theory. European Journal of Operational Research, v. 200, n. 3, p. 812-821, 2010. http://dx.doi.org/10.1016/j. ejor.2009.01.016

CASAROTTO FILHO, N.; PIRES, L. H. Redes de pequenas e médias empresas e desenvolvimento local: estratégias para a conquista da competitividade global com base na experiência italiana. 2 ed. São Paulo: Atlas, 2001.

COSTA, H. G.; MOTTA, S. S.; GUTIERREZ, R. H. Avaliação da produção docente: abordagem multicritério pelo método ELECTRE II. In: ENCONTRO NACIONAL DE ENGENHARIA DE PRODUÇÃO, 26., 2006, Fortaleza. Anais...

EDWARDS, W. How to use multiattribute utility measurement for social decision making. IEEE Transactions on Systems, Man and Cybernetics, v. 7, n. 5, p. 326-340, 1997.

FIGUEIRA, J. et al. Electre Tri-C, a multiple criteria decision aiding sorting model applied to assisted reproduction. International Journal of Medical Informatics, v. 80, n. 4, p. 265-273, 2005.

FREITAS, A. L, P. F.; RUBIM, A. V.; MANHÃES, N. R. C. Emprego do método ELECTRE III na seleção de equipamentos. In: ENCONTRO NACIONAL DE ENGENHARIA DE PRODUÇÃO, 24., 2004, Florianópolis. Anais..

GEORGOPOULOU, E.; LALAS, D.; PAPAGIANNAKIS, L. A multicriteria decision aid approach for energy planning problems: the case of renewable energy option. European Journal of Operational Research, v. 103, n. 1, p. 38-54, 1997.

GIANNOULIS, C.; ISHIZAKA, A. A web-based decision support system with ELECTRE III for a personalised ranking of British universities. Decision Support Systems, v. 48, n. 3, p. 488-197, 2010. http://dx.doi. org/10.1016/j.dss.2009.06.008

GOMES, L. F. A. M. Teoria da decisão. São Paulo: Thomson, 2007. 
GOMES, L. F. A. M.; GOMES, C. F. S.; ALMEIDA, A. T. Tomada de decisão gerencial: enfoque multicritério. São Paulo: Atlas, 2002.

HOKKANEN, J.; SALMINEN, P. Choosing a solid waste management system using multi criteria decision analysis. European Journal of Operational Research, v. 98, n. 1, p. 19-36, 1997. http://dx.doi. org/10.1016/0377-2217(95)00325-8

HUGONNARD, J.; ROY, B. Ranking of suburban line extension projects for the Paris metro system by multicriteria method. Transportation Research, v. 16, n. 4, p. 301-31, 1984.

LIANG, G.; WANG, M. A Fuzzy multi-criteria decision-making method for facility site selection. International Journal of Production Research, v. 29, n. 11 , p. 2313-2330, 1991. http://dx.doi. org/10.1080/00207549108948085

MENDONÇA, F. M. Formação, desenvolvimento e estruturação de arranjos produtivos locais em Minas Gerais. São Paulo: Blucher, 2010.

MOREIRA, R. A. Análise multicritério dos projetos do SEBRAE/RJ através do Eletre IV. 2007. Dissertação (Mestrado) - Instituto Brasileiro de Mercado de Capitais - IBMEC, Rio de Janeiro, 2007.

OLIVEIRA JÚNIOR, H. A lógica difusa: aspectos práticos e aplicações. Rio de Janeiro: Interciência, 1999.

PEREIRA, C. G. Análise de crédito bancário: um sistema especialista com técnicas difusas para os limites da agência. Dissertação (Mestrado) - Universidade Federal de Santa Catarina - UFSC, Santa Catarina, 1995.
ROGER, M.; BRUEN, M. Choosing realistic values of indifference, preference and veto thresholds for use with environment criteria with ELECTRE. European Journal of Operational Research, v. 107, n. 3, p. 542-551, 1998. http://dx.doi.org/10.1016/S0377-2217(97)00175-6

ROGER, M.; BRUEN, M.; MAYSTRE, L. Electre and decision support. New York: Kluwer Academic Publishers, 2000. http://dx.doi. org/10.1007/978-1-4757-5057-7

ROY, B.; BOUYSSOU, D. Aide multicritère à la décision: méthodes et cas. Paris: Economica, 1993.

ROY, B. Multicriteria methodology for decision aiding. London: Kluwer Academic Publishers, 1996. http:// dx.doi.org/10.1007/978-1-4757-2500-1

ROY, B. ELECTRE III: un alghoritme de methode de classements fonde sur une representation floue des préférences en presence de critères multiples. Cahieres de CERO, v. 20, n. 1, p. 3-24, 1978.

SHANIAN, A.; SAVADOGO, O. ELECTRE I: decision support model for material selection of bipolar plates for polymer electrolyte fuel cells applications. Journal of New Materials for Electrochemical Systems, v. 9, n. 3, p. 191-199, 2006.

SHIMIZU, T. Decisão nas organizações. 2. ed. São Paulo: Atlas, 2006.

VINCKE, P. H. Multicriteria decision aid. Chichester: Wiley, 1992.

ZADEH, L. The concept of a linguistic variable and its applications to approximate reasoning. Information Sciences, v. 8, n.2, p.7-13, 1975. 